\title{
Two centuries of climate events detected in coral records from Ishigaki and Ogasawara Islands, Japan
}

\author{
Atsushi Suzukı \\ Geological Survey of Japan, National Institute of Advanced Industrial Science and Technology (AIST), Japan; a.suzuki@aist.go.jp

\begin{abstract}
Coral records from the subtropical northwestern Pacific reveal abrupt cooling and a shift toward fresher surface ocean conditions between 1900 and 1910 AD.
\end{abstract}

O nly about 100 years or less of observational data for the ocean environment in the subtropical northwestern Pacific exist. Geochemical tracers, such as stable oxygen isotopes $\left(\delta^{18} \mathrm{O}\right)$, strontium/calcium $(\mathrm{Sr} / \mathrm{Ca})$ and uranium/calcium (U/Ca) ratios in skeletons of massive corals are excellent paleoclimatic and paleoceanographic proxies to extend our knowledge of the ocean environment beyond the instrumental record. Recently, a coral record going back to $1873 \mathrm{AD}$ from Chichijima in the Ogasawara Islands (Felis et al. 2009) and a 165-year long record from Ishigaki Island, southern Ryukyus (Mishima et al. 2010) have become available (Fig. 1). These coral records reveal several coupled oceanatmosphere phenomena during the early $20^{\text {th }}$ century in the northwestern subtropical Pacific.

\section{Abrupt freshening event recorded in the Ogasawara coral}

The Ogasawara Islands $\left(27^{\circ} \mathrm{N}, 142^{\circ} \mathrm{E}\right)$, approximately $1000 \mathrm{~km}$ south of Tokyo, Japan, are exposed to the open ocean environment. Felis et al. (2010) reported that the winter $\mathrm{Sr} / \mathrm{Ca}$ and U/ Ca paleotemperature records from an annually banded coral were significantly correlated with the instrumental winter Pacific Decadal Oscillation (PDO) index over the last century. The PDO is an indicator of North Pacific sea surface temperature (SST) anomalies poleward of $20^{\circ} \mathrm{N}$.

Felis et al. (2009) also reconstructed annually resolved sea surface salinity (SSS) variations since 1873 from the $\delta^{18} \mathrm{O}$ record and identified an abrupt shift toward fresher surface ocean conditions between 1905 and 1910 (Fig. 2C). They concluded that the Ogasawara freshening could not be explained by precipitation changes but resulted from a combination of atmospheric and oceanic convection processes.

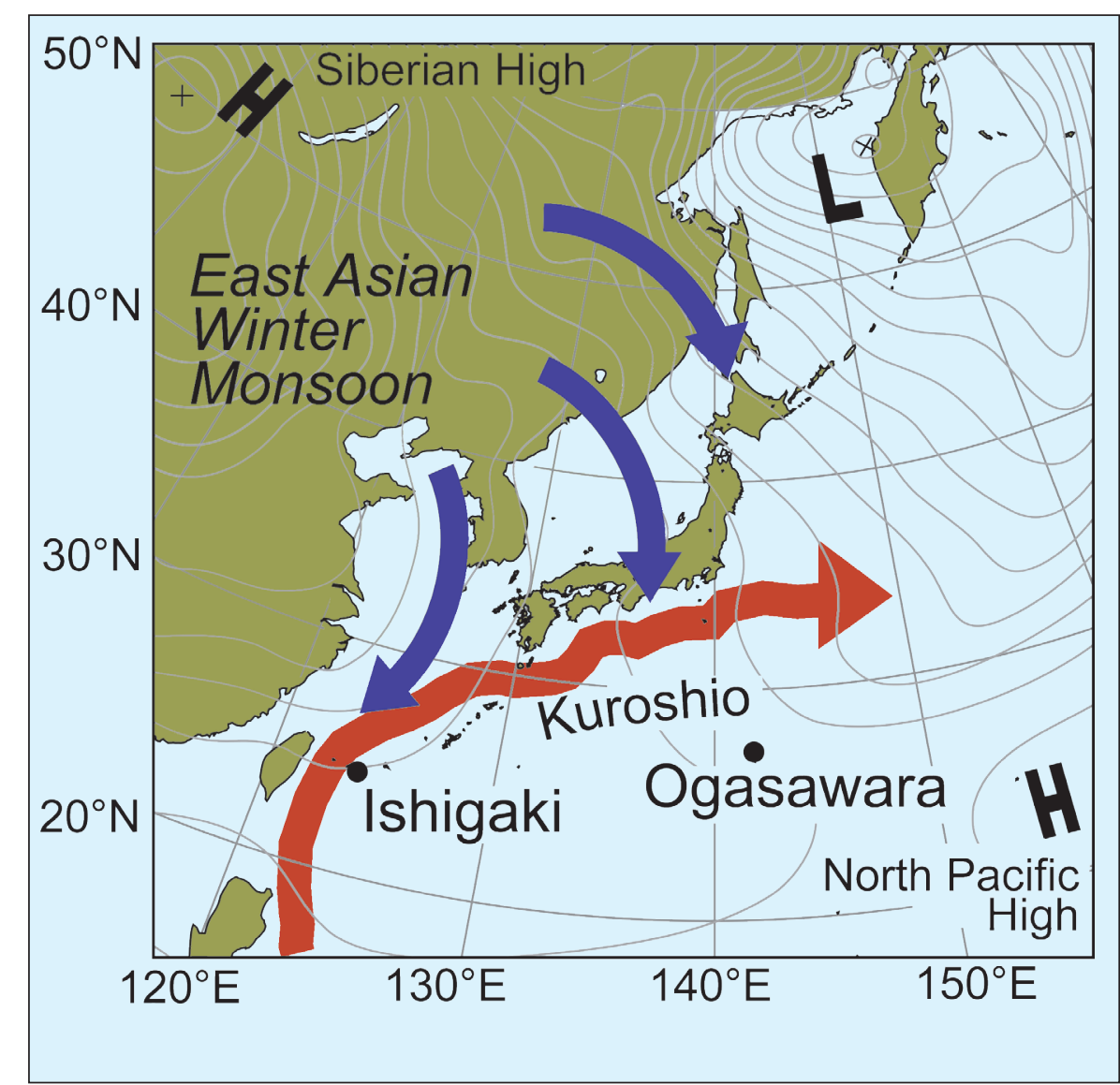

Figure 1: Map of the northwestern Pacific showing the locations of the Ishigaki and Ogasawara Islands. The typical winter East Asian sea-level atmospheric pressure distribution (isolines) and the path of the Kuroshio current (red arrow) are also shown. Northerly winds (East Asian winter monsoon) blowing from the Siberian High influence the climate around Japan in winter (blue arrows). Modified from Mishima et al. (2010).

\section{The Ishigaki coral record and the East Asian winter monsoon}

Ishigaki Island $\left(24^{\circ} \mathrm{N}, 124^{\circ} \mathrm{E}\right)$ is in the marginal East China Sea (Fig. 1), where the ocean environment reflects complex influences from the Kuroshio Current and the East Asian monsoon. Although $\delta^{18} \mathrm{O}$ can record changes in both SST and SSS, at Ishigaki, seasonal SSS changes are very small, whereas seasonal SST are well reflected by the $\delta^{18} \mathrm{O}$ values. Winter $\delta^{18} \mathrm{O}$ and observed SST, measured at Ishigaki Port, showed a significant linear correlation (Fig. $2 A, B)$, which suggests that the winter $\delta^{18} \mathrm{O}$ data from this coral sample is a good proxy for reconstructing past climate change. SST reconstructions can be used to investigate the relationship between the East Asian winter monsoon (EAWM) and the EI Niño-Southern Oscillation (ENSO). Before 1988/1989, the coral-based and instrumental winter SSTs were related to the EAWM, whereas after that winter they were more highly correlated to ENSO, reflecting a regional change in the climate regime (Tsunoda et al. 2008).

The Ishigaki $\delta^{18} \mathrm{O}$ coral record revealed abrupt cooling during 19001905 (Fig. 2A). This cold event was also seen in the coral $\mathrm{Sr} / \mathrm{Ca}$ data, and its timing is consistent with Japanese observations of exceptionally low temperatures in the winter of 1902. The presence of a strong Siberian High coupled with a strong EAWM and weak 


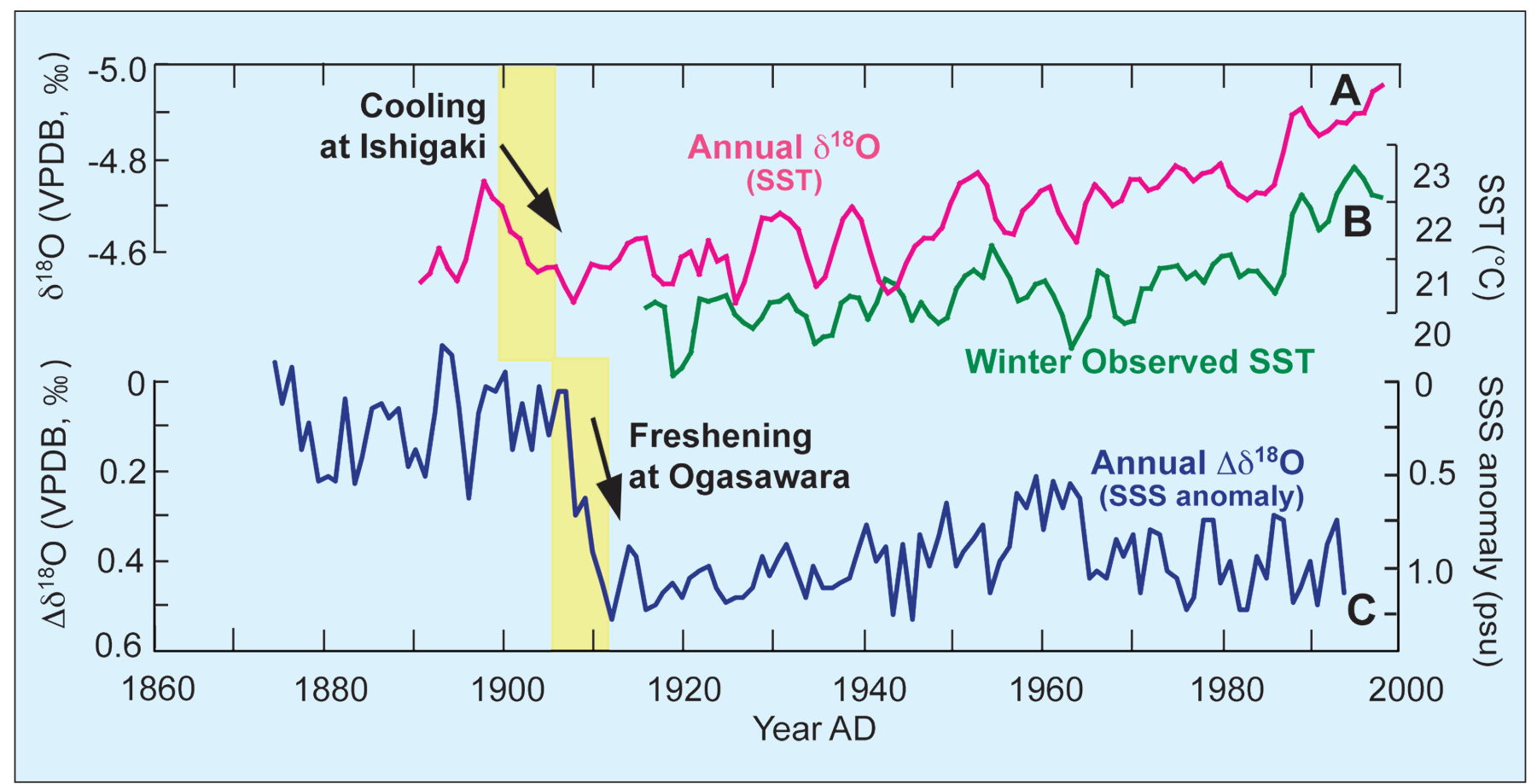

Figure 2: Time series of (A) annual coral $\delta^{18} \mathrm{O}(\mathrm{SST})$ data, (B) observed winter (December-February average) SST at Ishigaki Island, and $(\boldsymbol{C})$ residual coral $\delta^{18} \mathrm{O}$ records ( $\triangle \delta^{18} \mathrm{O}$ ) in the Ogasawara Islands, based on both $\delta^{18} \mathrm{O}$ and Sr/Ca.VPDB refers to the Vienna PeeDee Belemnite standard. The coral-based sea surface salinity (SSS) anomaly was calculated from the regional $\delta^{18} \mathrm{O}$ seawater-salinity relationship (0.42\%o per 1 psu; Felis et al. 2009). The yellow bars correspond to the abrupt cooling (1900-1905) inferred at Ishigaki Island and the abrupt freshening (1905-1910) in the Ogasawara Islands.

westerly winds, possibly accompanied by active heat convection in the tropics, might account for the cold winter at this time (Mishima et al. 2010).

\section{Unique observations of coupled early $20^{\text {th }}$ century climate events in the northwestern Pacific}

Interestingly, the abrupt cooling at Ishigaki Island occurred several years before the abrupt freshening in the Ogasawara Islands inferred from the coral isotope and trace element variations (Felis et al. 2009). The North Pacific High, which dominates the North Pacific Ocean, affects SSS; a strong North Pacific High is associated with high salinity. Strong westerly winds tend to enhance the North Pacific High because of its great height, approaching the height of the jet stream. Conversely, the freshening at Ogasawara suggests weak westerly winds, possibly associated with a weakened Kuroshio recirculation gyre, which flows from south of Honshu, Japan's main island, toward the Ogasawara Islands. In contrast, the Kuroshio's path from Taiwan to Ishigaki Island is relatively stable. Thus Ishigaki tends to be always under the influence of the saline Kuroshio and is thus unlikely to experience freshening such as that inferred at Ogasawara.

The decrease in SST indicated in the Ishigaki coral $\delta^{18} \mathrm{O}$ record was not observed in the Ogasawara coral record. A strong EAWM, which brings cold winds to the Japanese archipelago, is not likely to affect more pelagic environments such as the Ogasawara Islands. The approximately five-year time lag between the cold SSTs at Ishigaki and the freshening in the Ogasawara Islands is consistent with the findings of Deser et al. (1999), who reported that changes in the Kuroshio and associated North Pacific Ocean circulation lagged westerly wind changes by four to five years. By this mechanism, the early $20^{\text {th }}$ century cooling recorded by the Ishigaki coral may be related to the surface ocean freshening record in the Ogasawara coral. Thus, these records are unique evidences of coupled ocean-atmosphere phenomena during the first decade of the $20^{\text {th }}$ century in the subtropical northwestern Pacific.

\section{Future research}

The reliability of paleoclimate reconstructions could be improved by applying the dual proxy approach, utilizing element ratios such as $\mathrm{Sr} / \mathrm{Ca}$ along with oxygen isotope data, to the Ishigaki coral record. This approach would allow independent reconstruction of SST and SSS variation and make it possible to determine whether cold events around Ishigaki Island are associated with successive regional climate regime shifts. Coral-based reconstructions are now being extended to different time windows, including the Little Ice Age, the midHolocene warm period (Yokoyama et al. 2011), the last glacial maximum (Mishima et al. 2009), and the mid-Pliocene Warming Period (Watanabe et al. 2011). Also, the investigation of Porites coral tsunami boulders is a promising approach for long-term paleoclimate reconstruction (Suzuki et al. 2008; Araoka et al. 2010). The robustness of Porites coral records has been tested in a series of laboratory culture experiments to provide practical guidelines for the interpretation of coral climate proxies (Suzuki et al. 2005; Omata et al. 2008, Hayashi et al. in press).

\section{Selected references}

Full reference list online under:

http://www.pages-igbp.org/products/newsletters/ref2012_2.pdf

Felis T et al. (2009) Geology 37: 527-530

Mishima M et al. (2010) Geophysical Research Letters 37, doi: 10.1029/2010GL043451

Suzuki A, Hibino K, Iwase A, Kawahata H (2005) Geochimica et Cosmochimica Acta 69: 4453-4462

Watanabe T et al. (2011) Nature 471: 209-211

Yokoyama Y et al. (2011) Geophysical Research Letters 38, doi: 10.1029/2010GL044231 